INFLAMMATORY BOWEL DISEASE

\title{
Anti-Saccharomyces cerevisiae and antineutrophil cytoplasmic antibodies as predictors of inflammatory bowel disease
}

\author{
E Israeli, I Grotto, B Gilburd, R D Balicer, E Goldin, A Wiik, Y Shoenfeld
}

Gut 2005;54:1232-1236. doi: 10.1136/gut.2004.060228

See end of article for authors' affiliations

Correspondence to

Dr E Israeli,

Gastroenterology Unit,

Department of Medicine,

Hebrew University-

Hadassah Medical Center,

POB 12000, Jerusalem,

91120 , Israel;

eran-i@bezeqint.net

Revised version received 12 January 2005

Accepted for publication

20 January 2005
Background and aims: Several antibodies have been reported in the sera of patients with Crohn's disease (CD) and ulcerative colitis (UC). The most commonly described are anti-Saccharomyces cerevisiae mannan antibodies (ASCA) in CD and perinuclear antineutrophil cytoplasm antibodies (pANCA) in UC. Familial clustering of these antibodies has been described, suggesting they might be genetic markers. Our aim was to investigate the presence of these antibodies before the emergence of overt clinical manifestations.

Methods: Since 1980, the Israeli Defense Force (IDF) Medical Corps Serum Repository has stored serum samples obtained systematically from $5 \%$ of all recruits on enlistment, and from the same population on discharge from compulsory military service. We evaluated serum samples obtained from 32 subjects with $C D$ and eight with UC before they were clinically diagnosed, along with samples from matched controls. Results: ASCA were present in 10/32 (31.3\%) CD patients before clinical diagnosis compared with 0/95 $(0 \%)$ controls $(p<0.001)$. None of the eight patients with serum samples available before diagnosis of UC were ASCA positive. ASCA was positive in $54.5 \%$ of patients after diagnosis of CD. The mean interval between ASCA detection and diagnosis was 38 months. In $90 \%$ of patients, antibodies were detected in the first available serum sample; therefore, measurements of the average time from the presence of ASCA to diagnosis may be even longer. pANCA were present in $2 / 8(25 \%)$ patients with available sera before the diagnosis of UC. None of their 24 matched controls were positive $(p=0.014)$.

Conclusions: ASCA and pANCA may predict development of inflammatory bowel disease years before the disease is clinically diagnosed.
C rohn's disease (CD) and ulcerative colitis (UC) are common clinical subtypes of idiopathic inflammatory bowel disease (IBD). These diseases are characterised by excessive and tissue damaging inflammatory responses of the gastrointestinal tract. Although the aetiology is unknown, it is increasingly clear that these diseases represent the outcome of three essential interactive cofactors: environmental factors (for example, enteric microflora), multigenic host susceptibility, and immune mediated tissue injury. A variety of immune abnormalities have been described in IBD, both at the systemic and intestinal levels. Several autoantibodies differentially associated with CD and UC have been investigated in this respect. ${ }^{1}$ The most frequently studied in clinical trials, anti-Saccharomyces cerevisiae antibodies (ASCA) and perinuclear antineutrophil cytoplasmic antibodies (pANCA), have been suggested as diagnostic markers of the two diseases. The prevalence of these antibodies among patients with confirmed IBD has been well established. None the less, it is not known whether they may precede the clinical diagnosis of IBD.

We evaluated a prospectively assembled collection of frozen serum samples to test the hypothesis that the appearance of ASCA and pANCA precedes the diagnosis of IBD.

\section{METHODS}

\section{Serum samples}

Since 1980, the Israeli Defense Force (IDF) Military Corps Serum Repository has stored serum samples obtained from $5 \%$ of all recruits on enlistment, and from the same population on discharge from compulsory military service. The sample selection process was systematic, included both male and female soldiers, and was based on a numerical digit code derived from the subject's serial number. Samples were stored at $-20^{\circ} \mathrm{C}$.

The IDF computerised medical database was crosslinked with the serum repository records in order to identify military personnel diagnosed with CD or UC from 1980 to 2002. Records containing these diagnoses from 115 persons with sera in the repository were reviewed. Data on sex, ethnic group, date of birth, and date and age at diagnosis were obtained. Patients were excluded because of inadequate data, insufficient evidence of a diagnosis of IBD (see below), or absence of adequate serum samples. For each patient with at least one blood sample taken before IBD diagnosis, three controls were randomly selected from the repository records, matched for sex, age (within one year), and day of recruitment (for one of the CD cases only two controls were identified). For patients with a blood sample(s) taken after IBD diagnosis, two controls were randomly selected.

\section{Review of medical records}

The diagnosis of IBD was determined by review of medical records. Diagnostic criteria included typical clinical features of CD or UC and, in addition, at least one of the following: (a) characteristic endoscopic findings; (b) characteristic radiological features for CD in the small bowel; (c) macroscopic evidence of disease at laparoscopy; and (d) histopathological

Abbreviations: ASCA, anti-Saccharomyces cerevisiae antibody; pANCA, perinuclear antineutrophil cytoplasmic antibody; CD, Crohn's disease; UC, ulcerative colitis; IBD, inflammatory bowel disease; IDF, Israeli Defence Force; GMC, geometric mean concentration; SLE systemic lupus erythematosus; OR, odds ratio; PR3, proteinase 3; MPO, myeloperoxidase 
findings consistent with IBD. Patients with indeterminate colitis were excluded.

The protocol was reviewed and approved by the Human Use Committee of the IDF-Medical Corps. Informed consent for the testing of the coded stored serum samples and review of records by appropriate military personnel was waived. To protect the privacy of the patients, their names and unique personal information were not recorded or released. The date of sampling and analyses ranged from 1980 to 2002 .

\section{Antibody assays \\ ASCA testing}

Sera were analysed for ASCA expression in a blinded manner using a fixed IgG and IgA ELISA assay, Aeskulisa (Aesku Lab, Wendelsheim, Germany), according to the manufacturer's instructions. In short, $100 \mu \mathrm{l}$ of serum were added at a dilution of 1:101 to 96 well polystyrene microtitre plates. The plates are coated with highly purified mannan extracted from the yeast Saccharomyces cerevisiae. Bound ASCA was detected by incubation with horseradish peroxidase $\operatorname{IgG}$ or IgA conjugate (goat antihuman). Unbound conjugate was washed off, and addition of TMB substrate generated an enzymatic colorimetric (blue) reaction which was stopped by diluted acid (colour changes to yellow). Colour development is proportional to concentrations of antibody present in sera. Samples were read at $450 \mathrm{~nm}$ with a reference wavelength of $620 \mathrm{~nm}$ on a Anthes HT2 microtitre plate reader (Anthos; Labtech Instruments, Salzburg, Austria). Diluted human sera provided by the manufacturer served as internal controls. Six calibrators were used $(0,3,10,100,300 \mathrm{U} / \mathrm{ml})$. The assays were evaluated quantitatively by calculation of a standard curve from the six calibrators. A sample was considered positive when calculated at values $>15 \mathrm{U} / \mathrm{ml}$. For every sample, two analyses on the same plate were carried out. Sera exhibiting ASCA reactivity (IgG and/or IgA) exceeding the normal reference range were termed "ASCA positive".

\section{Antineutrophil cytoplasmic (atypical pANCA) testing}

Detection of pANCA was performed by an IIF technique on ethanol fixed buffy coat leucocytes, as previously described. ${ }^{2}$ Briefly, samples were initially diluted to $1: 20$ in phosphate buffered saline. An FITC conjugated rabbit antihuman IgG antibody (Dako, Copenhagen, Denmark) was used for detection of bound IgG antibodies. All slides were evaluated by two independent observers; in the event of a difference in opinion, a third observer was decisive. Staining patterns that were considered ANCA positive were: perinuclear (pANCA) and cytoplasmic (cANCA) staining of neutrophils. Samples that were scored positive were further analysed by ELISA for antiproteinase 3 (PR3) and antimyeloperoxidase (MPO) antibodies, as described previously. ${ }^{2}$ Samples that were found positive for PR3-ANCA and MPO-ANCA by ELISA were excluded. These pANCA positive samples, negative to PR3, MPO, and ANA, were considered as neutrophil specific autoantibodies without a known molecular target, as described in detail previously. ${ }^{3}$

\section{Statistical analysis}

Demographic characteristics of CD and UC patients were described according to mean age, sex, and origin. Origin was designated as Ashkenazi (European or American descent), Sephardic (Asian or African descent), or Israeli (at least three generations born in Israel).

Among CD patients in whom a prediagnostic serum sample was available, we calculated matched odds ratios (ORs) for having a positive ASCA test compared with controls. In order to evaluate the time from ASCA formation to diagnosis of CD, we determined the prevalence rate of ASCA positivity within three time frames: more than 60 months, 37-60 months, and 36 or less before $\mathrm{CD}$ diagnosis. In this analysis we included all serum samples available for each time frame (that is, patients with two serum samples before CD diagnosis were included twice in the analysis, unless both samples were from the same time frame). By comparing each group to controls, matched ORs for each time frame were calculated. Due to the fact that none of the controls was positive for ASCA, the OR was estimated by designating one positive control in each case. All OR, 95\% confidence intervals (CI), and p values of these analyses were calculated applying the Mantel-Hanzsel method for matched analysis. The geometric mean concentration (GMC) of ASCA for all CD patients was calculated for each time frame, and one way ANOVA was used to test for the significance of time trend. We further calculated mean time before diagnosis for all positive samples. In order to calculate the accrual of ASCA positivity, a Kaplan-Meier survival curve of all CD patients was plotted, beginning at 60 months before diagnosis up to 18 months after diagnosis. For this analysis, a patient with a negative result was considered to be negative up to that point, and was censored at the time of the last available serum sample if it was also negative. A patient with a positive result was considered to be positive from that time onwards (until 18 months after diagnosis). Median time for seropositivity was calculated. All results are expressed as mean (SEM).

\section{RESULTS}

\section{Patient population}

A diagnosis of $\mathrm{CD}$ was established in 37 military personnel, some formerly and some currently on active duty. Thirty eight serum samples were available from 32 of these patients before a diagnosis of $\mathrm{CD}$ was established. Of these 32 patients $88 \%$ were males, 47\% were Ashkenazi Jews, 44\% were Sephardic Jews, and 9\% were of Israeli descent. Mean (SEM) age at diagnosis was $24.8(0.9)$ years. The earliest available serum sample for each patient was obtained a mean of 59 (4.4) months before the diagnosis, with a maximal interval of 189 months to diagnosis. Eleven patients had postdiagnosis serum samples taken from 1 to 28 months after diagnosis.

Table 1 Detection of anti-Saccharomyces cerevisiae antibodies (ASCA) in different time frames before diagnosis in 32 Crohn's disease patients compared with controls

\begin{tabular}{|c|c|c|c|c|c|c|c|c|}
\hline \multirow[b]{2}{*}{ Time before diagnosis } & \multicolumn{3}{|c|}{ Cases } & \multicolumn{2}{|c|}{ Controls } & \multirow{2}{*}{$\begin{array}{l}\text { Matched OR } \\
(95 \% \mathrm{Cl})\end{array}$} & \multirow[b]{2}{*}{$p$ Value } & \multirow{2}{*}{$\begin{array}{l}\text { Estimated matched OR* } \\
(95 \% \mathrm{Cl})\end{array}$} \\
\hline & $n$ & Positive & $\%$ & $\bar{n}$ & Positive & & & \\
\hline$>60$ months & 13 & 2 & 15.4 & 39 & 0 & $\infty(0.563-\infty)$ & 0.014 & $6.00(0.54-66.17)$ \\
\hline $37-60$ months & 6 & 1 & 16.7 & 18 & 0 & $\infty(0.08-\infty)$ & 0.083 & $3.00(0.19-47.96)$ \\
\hline $1-36$ months & 19 & 7 & 36.8 & 56 & 0 & $\infty(4.10-\infty)$ & $<0.001$ & $21.00(2.58-170.69)$ \\
\hline All subjects before diagnosis & $32 \dagger$ & 10 & 31.3 & 95 & 0 & $\infty(6.73-\infty)$ & $<0.001$ & $30.00(4.27-1301.93)$ \\
\hline After diagnosis & 11 & 6 & 54.5 & 22 & 0 & $\infty(2.63-\infty)$ & 0.001 & $12.00(1.44-99.68)$ \\
\hline
\end{tabular}

*The estimated matched odds ratio (OR) was calculated by designating one control in each group as being ASCA positive. $95 \% \mathrm{Cl}, 95 \%$ confidence interval. †Six of the 32 patients had two available serum samples before diagnosis and are therefore represented twice in the different time frames. 


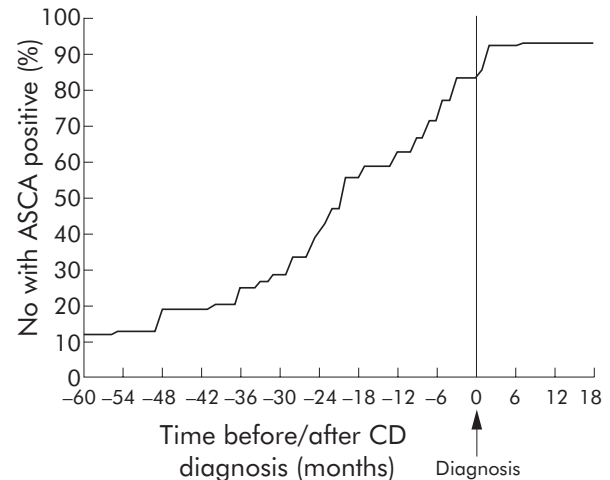

Figure 1 Kaplan-Meier product limit curve for the proportion of patients with positive anti-Saccharomyces cerevisiae antibodies (ASCA) relative to the time of diagnosis of Crohn's disease (CD).

A diagnosis of UC was established in 12 cases. Ten serum samples were available from eight of these patients, before diagnosis of UC was established. Of these 10 patients six were Ashkenazi Jews, one was Sephardic, and three were of Israeli descent. Mean (SEM) age at diagnosis was 23.5 (1.3) years. The earliest available serum sample for each patient was obtained a mean of 68 (17.7) months before the diagnosis, with a maximal interval of 134 months. Serum samples obtained after diagnosis (up to 53 months afterwards) were also available from six patients.

\section{ASCA prevalence in IBD patients and controls CD patients}

A total of 38 serum samples taken from 32 patients before diagnosis and 113 samples from matched controls were evaluated for ASCA antibodies. Ten of 32 (31.3\%) CD patients were positive for ASCA compared with 0/95 (0\%) controls (matched OR for having a positive test: cases $v$ controls $\infty$

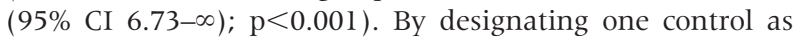
ASCA positive, the matched OR was 30.0 (95\% CI 4.271301.93). Of 11 patients with serum samples after diagnosis, six $(54.5 \%)$ were positive for ASCA. In all six cases in which ASCA was positive after diagnosis of CD, ASCA were also present in the prediagnostic serum samples.

\section{UC patients}

One of 12 UC patients was positive for ASCA (for this patient the serum sample was obtained after the diagnosis of the disease). One of 36 controls had a positive ASCA serum sample.

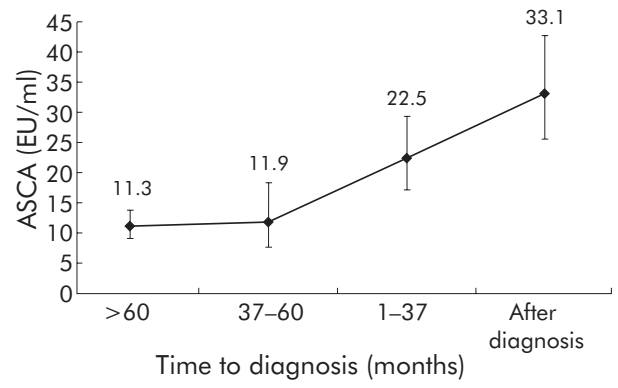

Figure 2 Geometric mean concentrations (SEM) of anti-Saccharomyces cerevisiae antibodies (ASCA) among all patients with Crohn's disease in different time frames.
Time from development of ASCA to diagnosis of CD

Table 1 describes the proportion of positive patients and controls with serum available within three time frames before and after diagnosis. The proportion of $\mathrm{CD}$ patients with positive ASCA increased from $15.4 \%$ at over 60 months before diagnosis to $36.8 \%$ within 36 months before diagnosis (54.5\% were positive after diagnosis). The OR for each time frame is also presented in table 1.

In nine of $10 \mathrm{CD}$ patients (90\%) with positive ASCA before diagnosis, antibodies were detected in the first available serum sample. The other CD patient was negative 80 months prior to diagnosis but tested positive at 48 months before diagnosis. Overall, the mean interval between ASCA detection and diagnosis was 38 months (range 20-77 months).

Figure 1 demonstrates accrual of patients testing positive in relation to the time of diagnosis. According to this analysis, $50 \%$ of CD patients were ASCA positive at 20 months before diagnosis. As mentioned, in a substantial proportion of cases, ASCA were present in the earliest available serum sample and were therefore never documented as having been undetectable. To allow for patients with positive ASCA in the first sample who may have been ASCA positive much earlier, we used the Kaplan-Meier product limit curve to evaluate the change in the proportion of patients with positive results over time.

Figure 2 demonstrates the GMC of ASCA at different time frames for all CD patients. GMC increased as the time of CD diagnosis was approached, and further increased in samples that were taken after diagnosis. This increase was statistically significant $(\mathrm{p}=0.029)$.

Table 2 depicts the evolution of ASCA levels in the subgroup of ASCA positive CD patients in whom sera were also available before diagnosis. As mentioned previously, ASCA were also present in all six cases before diagnosis. In 4/6 patients there was a significant increase in ASCA levels after diagnosis of disease compared with the prediagnostic serum sample. In one patient there was no change in levels (patient No 1) and in one patient (patient No 3) there was a decreased level after diagnosis.

\section{PANCA prevalence in IBD patients and controls UC patients}

Two of eight UC patients with available sera before diagnosis were positive for pANCA antibodies (25\%). None of their 24 matched controls were positive $(\mathrm{p}=0.014)$. For six patients, serum samples were available after diagnosis. Of these, one patient was positive for pANCA $(16.7 \%)$. None of the 12 matched controls was positive $(\mathrm{p}=0.157)$.

\section{$C D$ patients}

Two of $32 \mathrm{CD}$ patients with available serum samples before diagnosis were positive for pANCA (6.3\%) compared with

Table 2 Evolution of anti-Saccharomyces cerevisiae antibody (ASCA) titres in six ASCA positive Crohn's disease patients with available sera before diagnosis

\begin{tabular}{|c|c|c|c|c|}
\hline \multirow[b]{2}{*}{$\begin{array}{l}\text { Patient } \\
\text { No }\end{array}$} & \multicolumn{2}{|c|}{ Before diagnosis } & \multicolumn{2}{|c|}{ After diagnosis } \\
\hline & $\begin{array}{l}\text { Time } \\
\text { (months) }\end{array}$ & $\begin{array}{l}\text { ASCA } \\
\text { (EU/ml) }\end{array}$ & $\begin{array}{l}\text { Time } \\
\text { (months) }\end{array}$ & $\begin{array}{l}\text { ASCA } \\
\text { (EU/ml) }\end{array}$ \\
\hline 1 & -36 & 145.6 & +28 & 143.7 \\
\hline 2 & -22 & 52.5 & +1 & 103.5 \\
\hline 3 & -20 & 124.9 & +23 & 68.0 \\
\hline 4 & -16 & 16.2 & +1 & 23.6 \\
\hline 5 & -4 & 22.8 & +19 & 32.1 \\
\hline 6 & -4 & 30.4 & +1 & 46.2 \\
\hline
\end{tabular}


$0 / 95$ controls $(p=0.062)$. One of 11 patients with serum samples taken after diagnosis was positive $(9.1 \%)$ compared with none of 22 matched controls $(p=0.333)$.

\section{DISCUSSION}

It has recently been demonstrated in a variety of autoimmune diseases that the appearance of specific serum antibodies may precede the onset of clinical disease by many years. ${ }^{4}$ These antibodies may not be directly responsible for many of the manifestations of the disease, but are markers of future disease in presently healthy individuals. A partial list includes rheumatoid arthritis, ${ }^{5}$ systemic lupus erythematosus (SLE), ${ }^{6}$ and type I diabetes mellitus. ${ }^{7}$ In the case of SLE, a concept of a crescendo of autoimmunity culminating in clinical illness was formed. ${ }^{6}$ This concept is supported by data showing increasing concentrations of autoantibodies before diagnosis. ${ }^{68}$

The prospectively assembled IDF serum repository has provided an opportunity to examine the development of ASCA and pANCA before the onset of clinical illness in patients with IBD. ASCA were present in a subgroup of CD patients, years before the clinical onset of disease: $31.3 \%$ of patients were ASCA positive before clinical diagnosis and $54.5 \%$ after diagnosis versus none in controls. Overall, the mean interval between ASCA detection and diagnosis was 38 months (range 20-77). As in the majority of these subjects the first serum sample was ASCA positive, this means that the time interval was actually underestimated. In six patients who were ASCA positive after clinical onset, serum samples were also available before diagnosis. In all of these cases ASCA were present in prediagnostic serum samples.

Our findings correlate with the previously observed frequency of these antibodies in CD patients $(40-70 \%) .^{9-11}$ Despite modest sensitivity, several studies have found ASCA expression (either IgA or IgG) to be nearly 95\% specific for CD. ${ }^{9} 1112$

The reason for generation of ASCA remains unclear. ASCA are detected through their reactivity with sequences of mannose residues expressed in the cell wall mannan of $S$ cerevisiae. ${ }^{13}$ It was hypothesised that increased permeability in the small bowel of CD patients might lead to increased exposure of yeast antigens (which are a resident part of the normal intestinal flora) to immune reactive cells. Increased permeability of the small bowel as an early event in the pathogenesis of CD (before gross inflammatory damage to the bowel wall is apparent) may also explain our findings of the early appearance of ASCA. However, no association was found between ASCA titres and permeability of the small intestine, as measured by the cellobiose/mannitol test, ${ }^{14}$ or ${ }^{51} \mathrm{Cr} /$ EDTA. ${ }^{15}$ Thus ASCA and increased permeability of the small bowel are most likely independent phenomena in CD. ${ }^{16}$

ASCA have also been described as a genetic (or a familial) marker in IBD. This is based on studies demonstrating ASCA positivity in $20-25 \%$ of unaffected first degree relatives of patients with $\mathrm{CD} .{ }^{16-18}$ It was argued that whether the presence of ASCA in these healthy relatives is genetically determined or attributed to an environmental factor, it does not have any clinical implication. ${ }^{1}$ As none of the studies provided long term follow up on these subjects, no conclusion can be made as to whether they actually remain unaffected in years to come. We found that ASCA levels increased in asymptomatic subjects as the time to diagnosis of CD approached (fig 2). Therefore, the question relating to the clinical importance of the presence of ASCA in an asymptomatic family member may only be resolved by prospective studies with follow up of ASCA titres, as well as clinical symptoms in these individuals.

Recent evidence suggests that ASCA serology may correlate with particular clinical features, including young age at onset of disease, ${ }^{16}$ ileal involvement, ${ }^{919}$ and development of strictures and fistulae. ${ }^{20}$ Furthermore, ASCA have also been associated with increased risk for early surgery (defined as occurring within three years of diagnosis). ${ }^{21}{ }^{22}$ Early identification of a rising titre of ASCA may help to identify a subgroup of patients that might benefit from a more aggressive approach with preventive medical therapy or immunomodulation. This may alter the course of disease and decrease the necessity for surgery. ${ }^{22}$

Should asymptomatic persons incidentally discovered to be ASCA positive be monitored? Based on our findings it can be argued that the presence of ASCA in asymptomatic subjects may be in itself a predictor for future development of CD, with the distinct pattern of clinical features, as described above. This would be especially relevant for high risk individuals, such as asymptomatic first degree family members of $\mathrm{CD}$ patients. None the less, it is too early to recommend a specific course of action until further data are obtained through prospective clinical trials.

We were able to identify a relatively small number of UC patients with available serum samples before diagnosis of disease. pANCA were present in $25 \%$ of UC patients before clinical diagnosis compared with none of the controls. In our small cohort, only $1 / 6$ patients $(16.7 \%)$ were pANCA positive after diagnosis. The accuracy of pANCA as a diagnostic tool largely depends on the technique employed. ${ }^{323} \mathrm{~A}$ wide range of sensitivities (from $0 \%$ to $63 \%$ ) has been reported ${ }^{24}$ although, as in the case of ASCA for CD patients, the specificity of pANCA for diagnosis of UC is high.

ANCA constitute a heterogenous group of antibodies which are mainly directed against constituents of neutrophil granules in patients with primary vascultides. The neutrophil specific pANCA-like antibodies found in the sera of patients with UC however seem to target many different neutrophil antigens located in nuclei, granules, and cytosol. ${ }^{3}$ In UC, serum pANCA are thought to reflect mucosal pANCA production, ${ }^{25}$ thus implying that recognition of mucosal antigen(s) is involved. Most studies do not support a relationship between the presence or level of pANCA and UC activity, ${ }^{26}$ and pANCA persist after total colectomy. ${ }^{27}$ Although our study reports only a small number of UC patients, it clearly demonstrates that pANCA is present in a subgroup of patients before clinical diagnosis.

In summary, our results demonstrate that clinical IBD is preceded by the presence of specific antibodies for many years before diagnosis. In the case of CD, we found a steady increase in the percentage of ASCA positive cases as well as a significant rise in levels of ASCA as time progresses, until symptoms of clinical illness appear. This distinct pattern, which is a common feature of autoimmune disease, undermines the current belief that ASCA are simply a genetic marker of CD.

Thus the presence of ASCA in a high risk healthy individual might be a marker for future development of $\mathrm{CD}$ and may even predict the clinical course. A similar association may exist for pANCA and UC.

\footnotetext{
Authors' affiliations

E Israeli, Gastroenterology Unit, Department of Medicine, Hebrew University-Hadassah Medical Center, Jerusalem, Israel, and IDF Medical Corps

I Grotto, R D Balicer, IDF Medical Corps

B Gilburd, Department of Medicine ' $B$ ' and Center for Autoimmune Diseases, Chaim Sheba Medical Center, Sackler Faculty of Medicine, Tel-Aviv University, Israel

E Goldin, Gastroenterology Unit, Department of Medicine, Hebrew University-Hadassah Medical Center, Jerusalem, Israel

A Wiik, Statens Serum Institute, Department of Autoimmunology, Copenhagen, Denmark
} 
Y Shoenfeld, Department of Medicine ' $B$ ' and Center for Autoimmune Diseases, Chaim Sheba Medical Center, and Incumbent of Laura Schwarz-Kipp for Research of Autoimmune Disease, Sackler Faculty of Medicine, Tel-Aviv University, Israel

Conflict of interest: None declared.

\section{REFERENCES}

1 Reumaux D, Boualem S, Poulain D, et al. Serological markers in inflammatory bowel diseases. Best Pract Res Clin Gastroenterol 2003:17:19-35.

2 Wiik A. Antineutrophil cytoplasmic antibodies (ANCAs) and ANCA testing. In Rose NR, Hamilton RG, Detrick B, eds. Manual of clinical laboratory immunology, 6th edn. Washington DC: ASM Press, 2002:981-6.

3 Wiik A. Neutrophil-specific autoantibodies in chronic inflammatory bowel diseases. Autoimmunity Rev 2002;1:67-72.

4 Scofield RH. Autoantibodies as predictors of disease. Lancet 2004;363:1544-6.

5 Nielen MM, van Schaardenburg D, Reesink HW, et al. Specific autoantibodies precede the symptoms of rheumatoid arthritis: a study of serial measurements in blood donors. Arthritis Rheum 2004;50:380-6.

6 Arbuckle MR, McClain MT, Rubertone MV, et al. Development of autoantibodies before the clinical onset of systemic lupus erythematosus. N Engl J Med 2003:349:1526-33.

7 LaGasse JM, Brantley MS, Leech NJ, et al. Washington State Diabetes Prediction Study. Successful prospective prediction of type 1 diabetes in schoolchildren through multiple defined autoantibodies: an 8-year follow-up of the Washington State Diabetes Prediction Study, Diabetes Care 2002;25:505-11.

8 Arbuckle MR, James JA, Kohlhase KF, et al. Development of anti-dsDNA autoantibodies prior to clinical diagnosis of systemic lupus erythematosus. Scand I Immunol 2001;54:21 1-19.

9 Quinton JF, Sendid B, Reumaux D, et al. Anti-Saccharomyces cerevisiae mannan antibodies combined with antineutrophil cytoplasmic autoantibodies in inflammatory bowel disease: Prevalence and diagnostic role. Gut 1998;42:788-91.

10 Ruemmele FM, Targan SR, Levy G, et al. Diagnostic accuracy of serological assays in pediatric inflammatory bowel disease. Gastroenterology 1998.115.822-9.

11 Sendid B, Colombel F, Jaquinot PM, et al. Specific antibody response to oligomannosidic epitopes in Crohn's disease. Clin Diagn Lab Immunol 1996;3:219-26.

12 Hoffenberg EJ, Fidanza S, Sauaia A. Serological testing for inflammatory bowel disease. J Pediatr 1999;134:447-52.
13 Heelan BT, Allan S, Barnes RM. Identification of a 200-kDa glycoprotein antigen of Saccharomyces cerevisiae. Immunol Lett 1991;28:181-5.

14 Secondulfo $M$, de Magistris L, Fiandra $R$, et al. Intestinal permeability in Crohn's disease patients and their first degree relatives. Digest Liver Dis 2001;33:680-5.

15 Peeters M, Geypens B, Claus D, et al. Clustering of increased small intestinal permeability in families with Crohn's disease. Gastroenterology 1997:113:802-7.

16 Vermiere S, Peeters M, Vlietinck R, Joossens et al. Anti-Saccharomyces cerevisiae antibodies, phenotypes of IBD, and intestinal permeability: A study in IBD families. Inflamm Bowel Dis 2001:7:8-15.

17 Annese V, Andreoli A, Andriulli A, et al. Familial expression of antiSaccharomyces cerevisiae Mannan antibodies in Crohn's disease and ulcerative colitis: a GISC study. Am J Gastroenterol 2001;96:2407-12.

18 Seibold F, Stich O, Hufnagl R, et al. Anti-Saccharomyces cerevisiae antibodies in inflammatory bowel disease: A family study. Scand J Gastroenterol 2001;36:196-201.

19 Vermiere S. Serological diagnosis in IBD. Inflamm Bowel Dis Monit 2002;3:82-9.

20 Vasiliauskas EA, Lam LY, Karp LC, et al. Marker antibody expression stratifies Crohn's disease into immunologically homogeneous subgroups with distinct clinical characteristics. Gut 2000;47:487-96.

21 Sastegni $\mathbf{R}$, Daperno M, Ercole $E$, et al. Detection of anti-Saccharomyces cerevisiae antibodies in Crohn's disease: is it a reliable diagnostic and prognostic marker. Dig Liver Dis 2001;33:755-61

22 Forcione DG, Rosen MJ, Kisiel JB, et al. Anti-Saccharomyces cerevisiae antibody (ASCA) positivity is associated with increased risk for early surgery in Crohn's disease. Gut 2004;53:1117-22.

23 MacDermott RP. Lack of current clinical value of serological testing in the evaluation of patients with IBD. Inflamm Bowel Dis 1999;5:64-5.

24 Sandborn WJ, Loftus EV Jr, Colombel JF, et al. Evaluation of serologic disease markers in a population-based cohort of patients with ulcerative colitis and Crohn's disease. Inflamm Bowel Dis 2001;7:192-201.

25 Targan SR, Landers CJ, Cobb L, et al. Perinuclear anti-neutrophil cytoplasmic antibodies are spontaneously produced by mucosal B cells of ulcerative colitis patients. J Immunol 1995; 155:2362-7.

26 Reumaux D, Colombel JF, Macy E, et al. Anti-neutrophil cytoplasmic autoantibodies (ANCA) in ulcerative colitis (UC): no relationship with disease activity. Inflamm Bowel Dis 2000;6:270-4.

27 Seibold F, Weber P, Klein R, et al. Clinical significance of antibodies against neutrophils in patients with inflammatory bowel disease and primary sclerosing cholangitis. Gut 1992;33:657-62.

\section{bmjupdates+}

bmiupdates+ is a unique and free alerting service, designed to keep you up to date with the medical literature that is truly important to your practice.

bmiupdates + will alert you to important new research and will provide you with the best new evidence concerning important advances in health care, tailored to your medical interests and time demands.

Where does the information come from?

bmiupdates+ applies an expert critical appraisal filter to over 100 top medical journals A panel of over 2000 physicians find the few 'must read' studies for each area of clinical interest

Sign up to receive your tailored email alerts, searching access and more... 